\title{
Density control of InP/GalnP quantum dots grown by metal-organic vapor-phase epitaxy
}

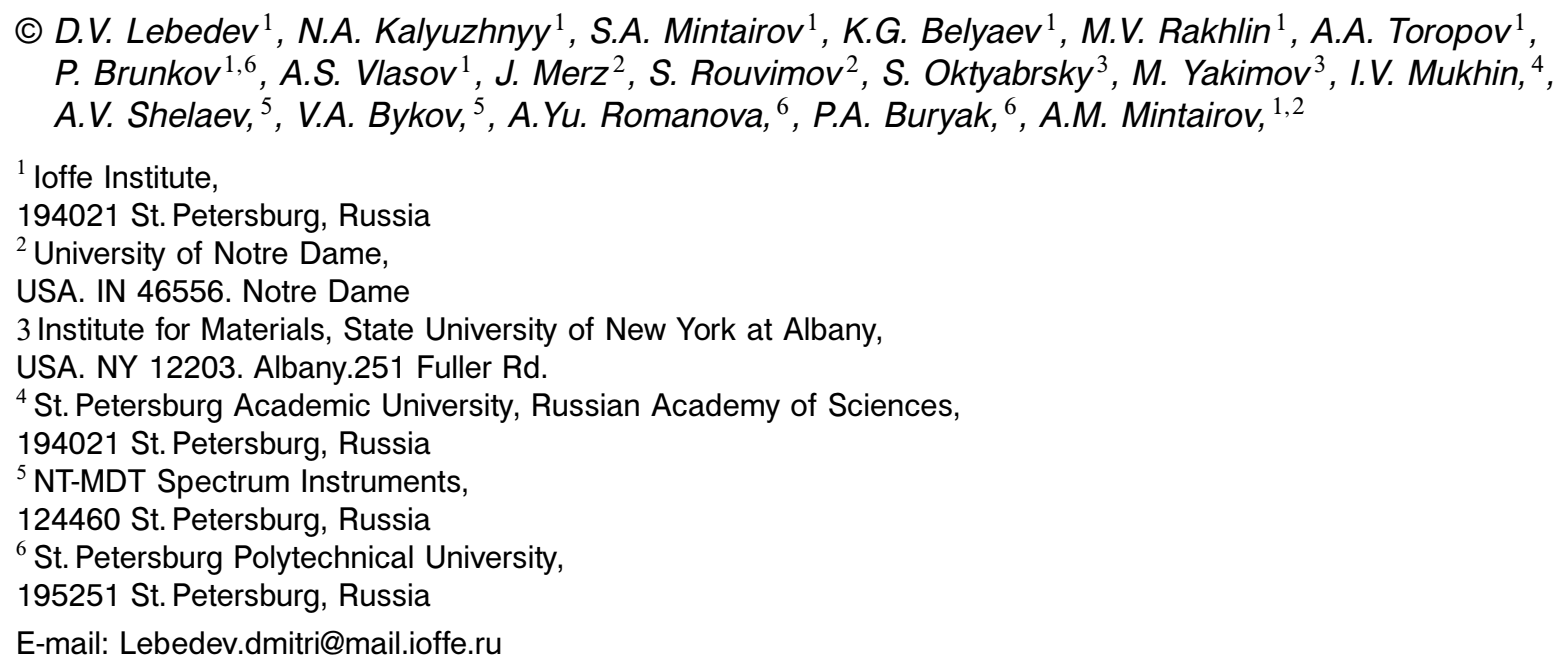

We investigated structural and emission properties of self-organized $\mathrm{InP} / \mathrm{GaInP}$ quantum dots (QD) grown by metal organic chemical vapor deposition using an amount of deposited In from 7 to 2 monolayers (ML). In the uncapped samples, using atomic force microscopy (AFM), we observed lateral sizes of $100-200 \mathrm{~nm}$, together with a bimodal height distribution having maxima at $\sim 5$ and $\sim 15 \mathrm{~nm}$, which we denoted as QDs of type $A$ and $B$, respectively; and reduction of the density of the type- $B$ dots from 4.4 to $1.6 \mu \mathrm{m}^{-2}$. The reduction of the density of $B$-type dots were observed also using transmission electron microscopy of the capped samples. Using single dot low-temperature photoluminescence (PL) spectroscopy we demonstrated effects of Wigner localization for the electrons accumulated in these dots.

\section{Acknowledgements}

A.M.M. and D.V.L. acknowledges support of the Ministry of Education and Science of the Russian Federation (contract 14.Z50.31.0021,7th April 2014) K.G.B., M.V.R. and A.A.T. gratefully acknowledge the financial support of the Russian Science Foundation (project 14-22-00107). 